\title{
Demonstration of duty cycle division multiplexing with bit error rate estimation
}

\begin{abstract}
Experimental demonstration of Duty Cycle Division Multiplexing (DCDM) is reported which validates the potential implementation of the system in communication field. We also presented the operation of bit error rate (BER) estimation algorithm, which is specially derived for DCDM. With the increase of link attenuation, the signal amplitude decreased and the pulses are distorted. BER estimation on the received signal was performed and measured against transmission distance of 100 meter until 400 meter copper wire. It is shown that the channel with the largest duty-cycle value shows the best performance. The experimental eye diagrams at several distances are also demonstrated and compared against their back-to-back counterpart.
\end{abstract}

Keyword: Multiplexing; Optical communication 